\title{
Systemic delivery of lentivirus-mediated secretable TAT-apoptin eradicates hepatocellular carcinoma xenografts in nude mice
}

\author{
JIN-LU MA ${ }^{1}$, SU-XIA HAN ${ }^{1}$, JING ZHAO $^{1}$, DAN ZHANG $^{1}$, LI WANG $^{1}$, YAO-DONG LI ${ }^{2}$ and QING ZHU ${ }^{1}$ \\ ${ }^{1}$ Department of Oncology, the First Affiliated Hospital of Xi'an Jiaotong University Medical College, Xi'an; \\ ${ }^{2}$ Department of General Surgery, the Affiliated Hospital of Xi'an Medical College, Xi'an, P.R. China
}

Received March 26, 2012; Accepted June 1, 2012

DOI: $10.3892 /$ ijo.2012.1547

\begin{abstract}
Apoptin, a chicken anemia virus-derived protein, has been shown to induce apoptosis in various human cancer cell lines, but not in normal cells, thus making it a candidate for the development of novel therapeutic strategies. To enable the efficient transduction of tumor cells with apoptin, we have developed a novel mammalian expression system for the secretion of apoptin in vitro. We have previously shown the efficient and tumor-specific killing of cells by adding a secretory signal peptide (SP) to the $\mathrm{N}$ terminus of transacting activator of transcription (TAT)-apoptin (SP-TAT-apoptin). In addition, our report showed the successful secretion of high levels of TAT-apoptin/GFP into the culture medium from HUVEC cells infected by lentivirus LV-SP-TAT-apoptin/GFP. To obtain sustained apoptin-induced tumor cell death in vivo, we injected the LV-SP-TAT-apoptin viruses via the tail vein for systemic delivery of the viruses; viruses expressing LV-SPTAT-GFP were used as a negative control. Markedly, almost all the hepatocellular carcinoma xenograft tumors disappeared following the treatment while the xenografts that received the control LV-SP-TAT-GFP viruses continued to grow. Moreover, the animal studies presented in this paper demonstrate a low toxicity of SP-TAT-apoptin in vivo, confirming and extending the results of the in vitro studies. Taken together, our data strongly suggest that systemic delivery of lentivirus-mediated secretable TAT-apoptin is feasible to eradicate liver cancer in vivo.
\end{abstract}

Correspondence to: Dr Qing Zhu, Department of Oncology, the First Affiliated Hospital, Xi'an Jiaotong University Medical College, Xi'an, P.R. China

E-mail: newzhuqing1972@yahoo.com

Abbreviations: TAT, transacting activator of transcription; PTD, protein transduction domain; SP, signal peptide; HCC, hepatocellular carcinoma; BFA, Brefeldin A; TUNEL, terminal deoxynucleotidyl transferase dUTP nick end labeling; H\&E, hematoxylin and eosin; PI, propidium iodide; PBS, phosphate buffered saline; DMEM, Dulbecco's modified Eagle's medium; FBS, fetal bovine serum; LSB, Laemmli sample buffer

Key words: apoptin, TAT-PTD, hepatocellular carcinoma, lentivirus, nude mice

\section{Introduction}

Apoptin, a chicken anemia virus-encoded protein, consists of 121 amino acids (1). An attractive feature of apoptin is that it induces apoptosis in a large number of human tumor or transformed cells but not in their normal, healthy counterparts $(2,3)$. This is correlated with the subcellular localization of the protein. In normal cells, apoptin is found in the cytoplasm whereas in tumor cells it is found in the nucleus (4-6). This suggests that apoptin may be used as an agent that selectively eliminates malignant cells, if it can be delivered into target cells in vivo in sufficient amounts. One straightforward strategy to reach this goal would be the application of the apoptin gene, rather than the protein itself.

The transacting activator of transcription (TAT) protein transduction domain (PTD) from human immunodeficiency virus type 1 (aa 47-57; YGRKKRRQRRR) is one of the most commonly used protein transduction systems $(7,8)$. In some studies, this TAT-PTD transduction domain has been successfully used to deliver apoptin into cancer cells in vitro. The data from these studies show that TAT-apoptin efficiently kills human tumor, but not normal cells (9-11). However, one disadvantage of using the TAT sequence in in vivo studies is that physical delivery of TAT fusion gene such as direct injection into the tumor bed is inefficient, as the produced recombinant protein may not reach all the cells within the tumor mass.

To enable the efficient transduction of tumor cells with apoptin, we have developed a novel mammalian expression system for the in vitro secretion of proteins $(12,13)$. We have previously shown the efficient and tumor-specific killing of cells by TAT-apoptin fused to a signal peptide (SP) whose amino acid sequence and corresponding cDNA sequences were generated by an SP hidden Markov model (SP-HMM) (14). This SP directs strong protein secretion and expression and the TAT-apoptin fusion protein secreted from transfected cells can re-enter the adjacent untransfected cells. It should be noted that in normal cells resistant to apoptin-mediated cell death, fusion protein is localized in the cytoplasm, whereas in sensitive cells it is in the nucleus. Nuclear localization was shown to be crucial for the cell-killing ability of apoptin (12). We have demonstrated that SP-TAT-apoptin induces apoptosis only in malignant cells, and its secretory property might greatly increase its potency, once it is delivered in vivo for cancer therapy. We recently generated a lentivirus-based 
vector to express SP-TAT-apoptin and apoptosis induced by apoptin was observed in human hepatocellular carcinoma (HCC) cells (13). We thus hypothesize that lentivirus-based SP-TAT-apoptin kills tumor cells; in addition, normal cells infected with the lentivirus-based SP-TAT-apoptin secrete TAT-apoptin fusion protein persistently, which leads to more extensive tumor cell death.

In this study, we investigated if Lentivirus-based delivery of SP-TAT-apoptin could serve as a therapeutic agent for HCC treatment in a mouse xenograft model. Our findings showed that systemic delivery of the Lentivirus-based SP-TAT-apoptin viruses eradicated xenograft tumors.

\section{Materials and methods}

Cell lines. Human immortalized liver cell line (HL-7702; catalog no. GNHu 6), and human HCC cell lines (HepG2; catalog no. TCHu 72 and Bel-7402; catalog no. TCHu 10) were obtained from the Shanghai Institutes for Biological Sciences (SIBS). Human Umbilical Vein Endothelia (HUVEC; catalog no. CRL-1730 ${ }^{\mathrm{TM}}$ ) cell line was obtained from American Type Culture Collection. Cells were cultured in Dulbecco's modified Eagle's medium (DMEM) supplemented with $10 \%$ fetal bovine serum (FBS), $50 \mu \mathrm{g} / \mathrm{ml}$ streptomycin and $100 \mu \mathrm{g} / \mathrm{ml}$ penicillin.

Constructs. GFP, apoptin, TAT-GFP, TAT-apoptin, SP-TATapoptin, and SP-TAT-GFP constructs contain the cDNA for GFP, apoptin, TAT-GFP, TAT-apoptin, SP-TAT-apoptin, and SP-TAT-GFP, respectively cloned into the pLenti6/V5-D$\mathrm{TOPO}^{\circledR}$ vector (catalog no. K4950-00, Invitrogen) (13).

Lentivirus ( $L V$ ) production. LV-SP-TAT-GFP and LV-SP-TATapoptin viruses were produced by Biomics Biotechnologies (Nantong, China) at a titer of $1.0 \times 10^{11}$ viral particle per ml, as described in our previous publication (13).

Transient transfection. HUVEC cells $\left(1.0 \times 10^{6}\right)$ per well were seeded in 6-well slides. The following day, cells were transfected with 600 ng DNA per well of the different constructs using Lipofectamine $^{\mathrm{TM}} 2000$ (catalog no. 11668-027, Invitrogen) according to the supplier's instructions. At 24 or $48 \mathrm{~h}$ after transfection, cells were fixed, permeabilized and apoptin/ GFP expression was examined by fluorescence microscopy. To confirm TAT fusion protein secretion by HUVEC cells transfected with these constructs, supernatant from the wells was centrifuged for $5 \mathrm{~min}$ at $200 \mathrm{~g}$ and three volumes of supernatant were mixed with one volume of $3 \mathrm{X}$ concentrated Laemmli sample buffer (3X LSB).

Stable transfection. HUVEC cells $\left(7 \times 10^{5}\right)$ were seeded in $10-\mathrm{cm}$ culture dishes and transfected the following day with $20 \mu \mathrm{g}$ of GFP, apoptin, TAT-GFP, TAT-apoptin, SP-TAT-apoptin, and SP-TAT-GFP constructs using Lipofectamine 2000 (catalog no. 11668-027, Invitrogen) according to the instructions. After 3 days, cells were put under selection with $8 \mu \mathrm{g} / \mathrm{ml}$ Blasticidin (catalog no. R21001, Invitrogen) after transfection with GFP, apoptin, TAT-GFP, TAT-apoptin, SP-TAT-apoptin, and SP-TAT-GFP constructs. Selection medium was changed every 3-4 days and clones were picked after 3 weeks. To examine
TAT-apoptin/GFP and SP-TAT-apoptin/GFP expression in each clone, $5 \times 10^{5}$ cells were seeded per well in 6-well slides. In order to confirm the expression of recombinant GFP, cells were fixed, permeabilized, counter stained with 4',6-diamino2-phenylindole (DAPI, catalog no. D9542, Sigma) containing mounting solution and examined by fluorescence microscopy (Olympus). The expression of recombinant apoptin was confirmed by immunoblot analysis using anti-V5 antibody (catalog no. R960-25, Invitrogen). After immunolabeling, cells were washed, stained with DAPI (catalog no. D9542, Sigma), and then viewed with fluorescent microscopy (Olympus). To confirm TAT fusion protein secretion, supernatant from HUVEC/TAT-GFP, HUVEC/SP-TAT-GFP, HUVEC/TATapoptin, and HUVEC/SP-TAT-apoptin clones was centrifuged for $5 \mathrm{~min}$ at $200 \mathrm{~g}$ and three volumes were mixed with one volume of 3X LSB. To check SP-TAT-apoptin secretion by HUVEC/SP-TAT-apoptin, supernatant was precipitated in $20 \%$ trichloroacetic acid for $10 \mathrm{~min}$ at $4^{\circ} \mathrm{C}$, centrifuged for $5 \mathrm{~min}$ at $13,000 \mathrm{rpm}$ and then the pellet was washed twice with acetone and dried at $95^{\circ} \mathrm{C}$ for 5-10 min. Finally, the pellet was lysed in $3 \mathrm{X}$ LSB and boiled for $5 \mathrm{~min}$.

Fluorescence microscopy. Cells were fixed in 4\% (wt/vol) paraformaldehyde in phosphate-buffered saline (PBS) for $3 \mathrm{~h}$, washed three times in PBS, permeabilized in $0.2 \%$ ( vol $/ \mathrm{vol})$ Triton X-100 in PBS for $15 \mathrm{~min}$ and then washed three times in PBS. For apoptin/GFP detection, nuclei were counterstained in medium containing DAPI. Cells were examined under a fluorescent microscope (Olympus).

Confocal microscopy. Cells were prepared as described for fluorescence microscopy and fluorescence images were recorded on an Olympus AX 70 (Olympus) confocal imaging system.

Flow cytometry assay. The sensitivity of cells to apoptosis was examined by fluorescence activated cell sorting (FACS) analysis using propidium iodide (PI) and fluorescein isothiocyanate (FITC) conjugated anti-Annexin V antibody according to the manufacturer's instructions (catalog no. 11858777001, Roche). Briefly, after incubating the cells for $48 \mathrm{~h}$ under normal glucose conditions or glucose-deprived conditions, the cells were stained with PI and FITC conjugated anti-Annexin $\mathrm{V}$ and then analyzed with a FACS Calibur (Coulter).

Western blot analysis. Cells were lysed in LSB $(62.5 \mathrm{mmol} / 1$ Tris- $\mathrm{Cl} \mathrm{pH}$ 6.7, $100 \mathrm{mmol} / \mathrm{l} \beta$-mercaptoethanol, $2 \%$ sodium dodecyl sulfate, $1 \mathrm{mg} / \mathrm{ml}$ aprotinin, $100 \mathrm{mg} / \mathrm{ml}$ phenylmethylsulphoxide). Equal amounts of proteins were subjected to SDS-PAGE and then western blotting for assessing GFP, TAT-GFP, SP-TAT-GFP, apoptin, TAT-apoptin, and SP-TAT-apoptin expression. Primary antibodies for GFP (catalog no. sc-9996) were purchased from Santa Cruz Biotechnology. $\beta$-actin blot served as protein loading control.

Concentration of supernatant. Two milliliters of supernatant from HUVEC, HUVEC/TAT-GFP, HUVEC/SP-TAT-GFP, HUVEC/TAT-apoptin, and HUVEC/SP-TAT-apoptin cells prepared for transduction were transferred to a vivaspin 2 column (catalog no. VS0291, Sartorius) which was centrifuged 
for $25 \mathrm{~min}$ at $2,000 \mathrm{~g}$ at $4^{\circ} \mathrm{C}$ resulting in a 20 -fold concentration. Target cells were treated for 30 min with $100 \mu$ l concentrated supernatant per well, fixed, permeabilized, and examined for apoptin/GFP expression by fluorescence microscopy. To assess those protein levels concentrated supernatant was mixed with 3X LSB and examined by western blot analysis.

$R T-P C R$. Total RNA was prepared using TRIzol ${ }^{\circledR}$ reagent (catalog no. 15596-018, Invitrogen) from xenograft tissues. To assess mRNA expression, RT-PCR was carried out using a PrimeScript RT-PCR Kit (catalog no. DRR014A, Takara). The primers for the apoptin gene and $\beta$-actin were described in our previous report (13). The amplification profile was as follows: $95^{\circ} \mathrm{C}$ for $45 \mathrm{sec}, 56^{\circ} \mathrm{C}$ for $45 \mathrm{sec}$, and $72^{\circ} \mathrm{C}$ for 1 min running in 30 cycles. After 30 amplification cycles, the expected PCR products were size fractionated onto a $1 \%$ agarose gel and stained with ethidium bromide.

Mouse xenografts and lentivirus injection. A total of 20 BALB/C null mice and 16 male ICR mice at 5 weeks of age were used for the experiment. The mice were subcutaneously injected in the upper portion of the hind limb with $10^{7}$ HepG2 cells. Three days after tumor cell engrafting, the mice were randomly divided into four groups of five mice each. When tumors were palpable ( $\sim 50 \mathrm{~mm}^{3}$ in 4-6 weeks), animals were treated once by one of the two routes: via the tail vein or intra-tumoral injection, with LV-SP-TAT-GFP or LV-SP-TATapoptin at a dose indicated in the figure legends. Tumor growth was monitored by measuring the length (L) and the width (W), and the volume was calculated by the formula of $\mathrm{V}=\mathrm{LxW}^{2} / 2$. The wet weight of dissected xenograft tumors was recorded at the end of the experiment. The protocols used in the study were approved by the Hospital's Protection of Human Subjects Committee.

Immunohistochemistry and TUNEL assay. For protein analysis, xenograft tumors were snap-frozen and stored at $-80^{\circ} \mathrm{C}$ before processing. For immunostaining analysis, specimens were fixed in $4 \%$ paraformadehyde, paraffin-embedded and 4-micron tissue sections were cut. Tumor sections were stained with hematoxylin and eosin (H\&E) to evaluate tumor structure. Primary antibody for V5 (catalog no. R960-25) was purchased from Invitrogen. Tissue sections were immunostained with primary antibody against V5 followed by exposure to FITC labeled secondary antibodies and by DAPI for nuclei (Sigma). Apoptotic cell death was determined by in situ TUNEL analysis with the In Situ Cell Death Detection Kit, POD (catalog no. 11684817910, Roche) as described in the specifications.

\section{Results}

Signal-peptide-linked TAT-apoptin is secreted, but not TAT-apoptin. To enable the efficient transduction of tumor cells with apoptin, we have developed a novel mammalian expression system for the in vivo secretion of proteins. Previously we designed a secretory TAT-apoptin fusion protein by adding a secretory signal SP to the N-terminus of the recombinant SP sequence (12). In this study, HUVEC cells were stably transfected with either TAT-apoptin/GFP or SP-TAT-apoptin/
GFP vectors, and the clones expressing TAT-apoptin/GFP or SP-TAT-apoptin/GFP were selected using Blasticidin $(8 \mu \mathrm{g} / \mathrm{ml})$. Fluorescence microscopy detected no difference with the different apoptin/GFP modifications (Fig. 1A). However, western blot analysis showed the SP-TAT-apoptin/GFP HUVEC stable clones secreted high levels of SP-TAT-apoptin/ GFP in the culture medium while the secretion from the TAT-apoptin/GFP HUVEC stable clones of TAT-apoptin/GFP was very low (Fig. 1B). Brefeldin A (BFA) is a fungal metabolite that inhibits protein transport out of the Golgi apparatus, causes disassembly of this organelle and eventually blocks the secretion of proteins from cells $(15,16)$. HUVEC cells expressing SP-TAT-apoptin/GFP were treated with different concentrations of BFA. As shown in Fig. 1C, secretion of SP-TAT-apoptin/GFP by HUVEC was significantly reduced with BFA treatment.

Lentivirus-based secretable TAT-apoptin is efficiently secreted and kills HCC cells in vitro. To further assess the secretory ability of SP-TAT-apoptin/GFP, lentiviruses LV-SPTAT-apoptin/GFP were generated as described in our previous publication (13). LV-SP-TAT-apoptin/GFP-infected HUVEC cells were examined for the secretion of TAT-apoptin/GFP in the supernatant by western blot analysis. High levels of recombinant protein were detected in the supernatant (Fig. 2A). Furthermore, apoptosis, estimated by flow cytometry assay (Annexin V and PI), was detected in HepG2 Bel-7402 cells treated with the concentrated supernatant of LV-SP-TAT-apoptin-infected HUVEC cells (41.22 and $50.31 \%$, respectively), in contrast to no induction of cell death in HepG2 and Bel-7402 cells treated with the concentrated supernatant of LV-SP-TAT-GFP-infected HUVEC cells. Moreover, there was no induction of cell death in HL-7702 cells treated with the concentrated supernatant of LV-SPTAT-apoptin/GFP-infected HUVEC cells (Fig. 2B). Using fluorescence microscopy, HepG2, Bel-7402 and HL-7702 cells treated with the concentrated supernatant of HUVEC cells infected with LV-SP-TAT-GFP for 30 min showed positive GFP expression in target cells. However, apoptin nuclear localization was detected only in HepG2 and Bel-7402 cells treated with the concentrated supernatant of HUVEC cells infected with LV-SP-TAT-apoptin for $30 \mathrm{~min}$, while apoptin nuclear localization was not detected in HL-77002 cells treated with the concentrated supernatant of HUVEC cells infected with LV-SP-TAT-apoptin for $30 \mathrm{~min}$ (Fig. 2C).

Lentivirus-based secretable TAT-apoptin eradicates HCC xenograft tumors in vivo after systemic delivery. A pilot experiment was initially carried out to determine a proper dose for efficient SP-TAT-apoptin expression in xenograft tumors. Xenograft tumors were generated in nude mice using the HCC cell line HepG2. When tumors were palpable (around $50 \mathrm{~mm}^{3}$ in size), 4 different doses (log-dilution, $2 \times 10^{6}-2 \times 10^{9}$ viral particles in $10 \mu \mathrm{l}$ total volume) of the recombinant LV-SPTAT-apoptin viruses were injected into the tumor (multiple sites per tumor). In addition, two other animals received the virus LV-SP-TAT-GFP $\left(2 \times 10^{9}\right.$ viral particles in $\left.10 \mu \mathrm{l}\right)$ as the negative controls. One week later, xenograft tumors were harvested for further analysis. RT-PCR and western blotting results showed a gradually increasing pattern in the apoptin 

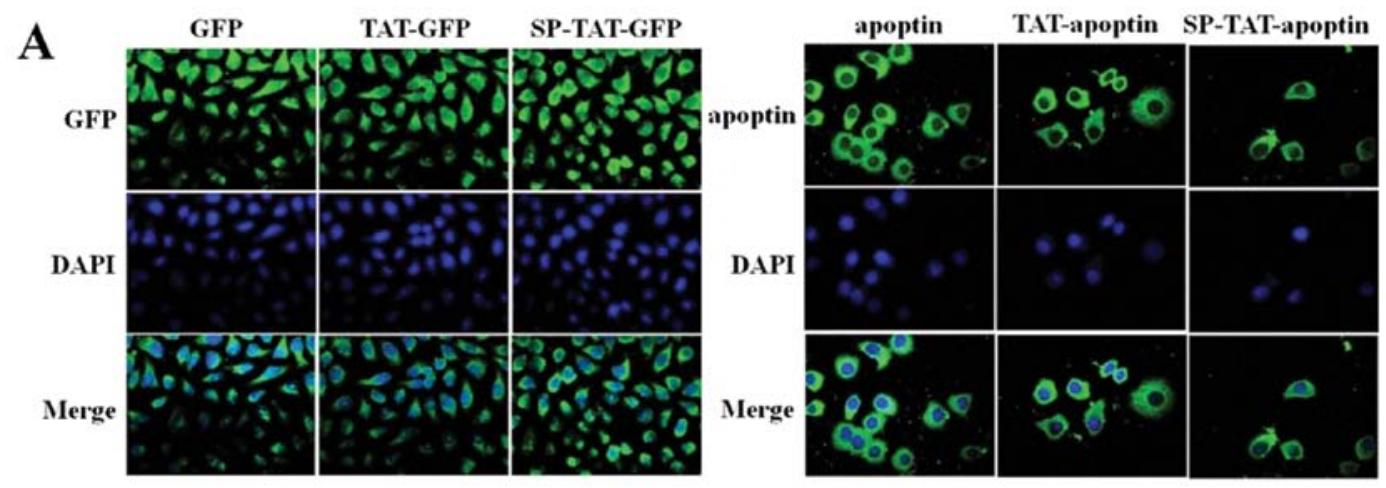

B

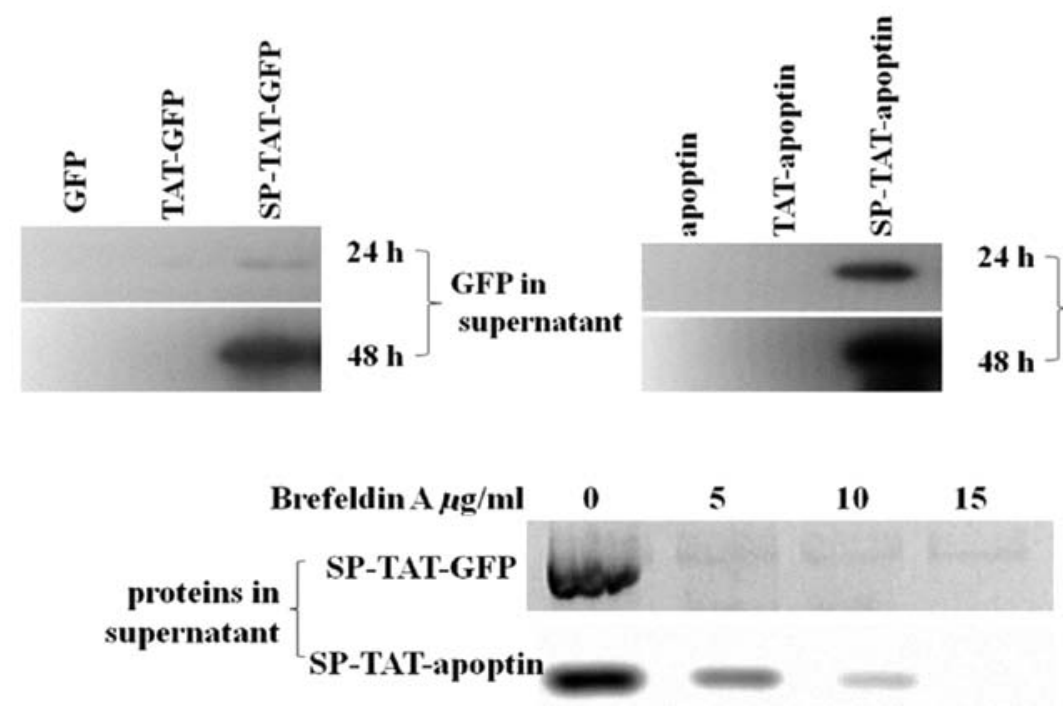

Figure 1. Apoptin/GFP expression in HUVEC/apoptin/GFP, HUVEC/ TAT- apoptin/GFP and HUVEC/SP- TAT-apoptin/GFP stable clones. (A) Fluorescence microscopy detected no difference with the different apoptin/ GFP modifications. Magnification, x600. (B) HUVEC/ SP-TAT-apoptin/GFP stable clones secreted high levels of TAT-apoptin/GFP in the culture medium while the secretions from HUVEC/ TAT- apoptin/GFP stable clones of TAT-apoptin/GFP and HUVEC/apoptin/GFP were very low. (C) The secretion of TAT-apoptin/GFP by HUVEC/SP-TAT-apoptin/GFP was significantly reduced by BFA treatment.

mRNA and protein levels (Fig. 3), reflecting a dose-dependent expression of viral genes. The peak effect was seen at the dose of $2 \times 10^{9}$ particles per $50 \mathrm{~mm}^{3}$.

Subsequently, we tested if the secretable TAT-apoptin could tackle the critical obstacle, HCC. HepG2 cells were injected into the upper portion of the hind limb of BALB/C null mice, and allowed to grow. Once HepG2 xenograft tumors were palpable $\left(\sim 50 \mathrm{~mm}^{3}\right)$, animals were randomly divided into two groups to receive an intra-tumoral injection of either LV-SP-TAT-GFP or LV-SP-TAT-apoptin viruses. Tumor growth was monitored for another 30 days. As shown in Fig. 4A and C, following intra-tumoral injection, LV-SPTAT-GFP virus-injected xenograft tumors grew constantly. By contrast, LV-SP-TAT-apoptin virus-injected xenograft tumors were completely suppressed. These data indicate that LV-SP-TAT-apoptin eliminated progression of HCC. At the end of the experiments, xenograft tumors were harvested for further analysis, including virus distribution (apoptin/ GFP expression) and apoptotic cell death (TUNEL assay). As shown in Fig. 4B and D, a diffused pattern of apoptin/ GFP expression was observed in virus-injected xenografts, although some spots displayed higher levels of apoptin/GFP expression, perhaps due to uneven distribution of injected viruses. In accordance with the tumor growth pattern, higher apoptotic index (TUNEL assay) was found in the LV-SP-TAT-apoptin virus-treated xenografts compared to the LV-SP-TAT-GFP virus-treated xenografts, which is consistent with our previous finding that LV-SP-TAT-apoptin virus leads to apoptotic cell death in HCC cells.

We showed that intra-tumoral injection of LV-SP-TATapoptin virus abrogated tumor growth but did not eradicate xenograft tumors. A plausible explanation might be the uneven distribution of the locally injected viruses, which affected the number of tumor cells (SP-TAT-apoptin producer cells) killed by TAT-apoptin. In order to achieve a better virus distribution and to induce a profound tumor recession, we went on to inject the viruses via the tail vein for systemic delivery of the viruses. Once HepG2 cell-based xenograft tumors were palpable $\left(\sim 50 \mathrm{~mm}^{3}\right)$ in nude mice, LV-SP-TAT-apoptin or LV-SPTAT-GFP viruses were injected via the tail vein at a dose of $2.0 \times 10^{9}$ viral particles per injection. Tumor growth and animal condition were monitored for one month. No animal presented any obvious complication and no visible side-effect was noticed due to the virus injection other than xenograft-related stress. As shown in Fig. 5A, LV-SP-TAT-GFP virus-injected tumor showed a rapid and continuous growth. However, in a group of eight animals that received LV-SP-TAT-apoptin injection, five tumors disappeared within 10 days and two other tumors 
A

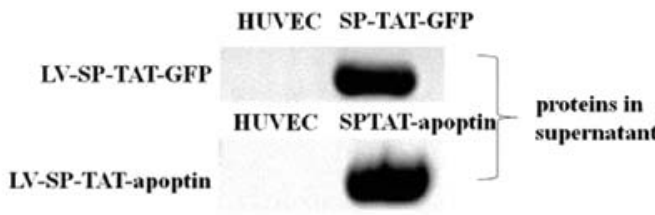

B

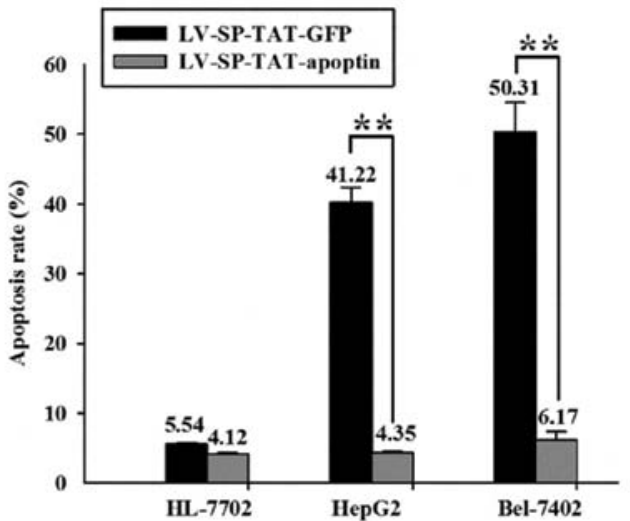

C

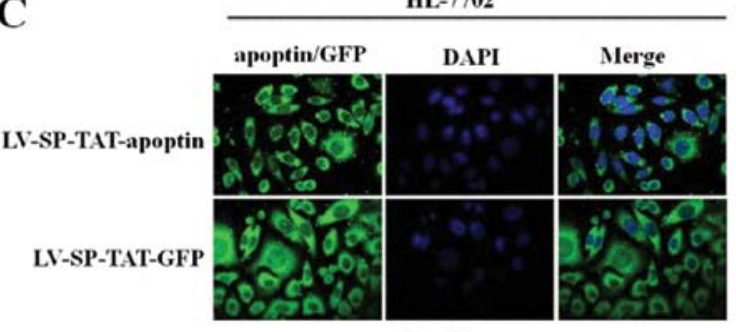

HepG2

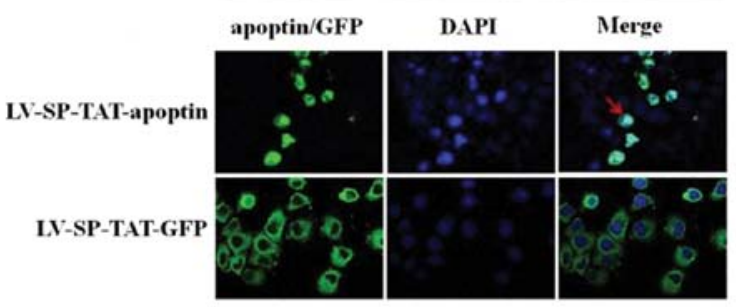

Bel-7402

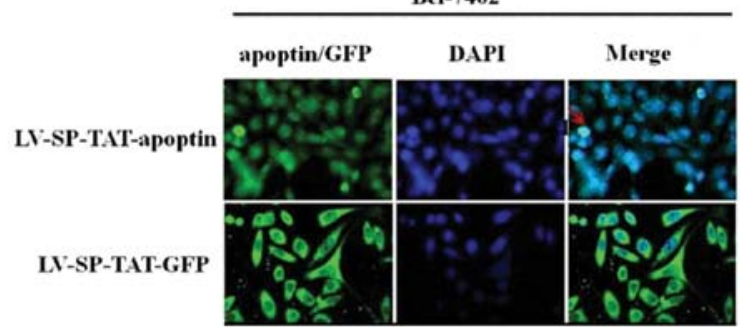

Figure 2. Lentivirus-based secretable TAT-apoptin is efficiently secreted and kills HCC cells. (A) LV-SP-TAT-apoptin/GFP-infected HUVEC cells expressing secretable TAT-apoptin/GFP were examined for the secretion of TAT-apoptin/GFP in the supernatant by western blot analysis. High levels of recombinant protein were detected in the supernatant. (B) Apoptosis, estimated by flow cytometry assay (Annexin V and PI) was detected in HepG2 Bel-7402 cells treated with the concentrated supernatant of LV-SP-TAT-apoptin-infected HUVEC cells (41.22 and 50.31\%, respectively), while there was no induction of cell death in HepG2 and Bel-7402 cells treated with the concentrated supernatant of LV-SP-TAT-GFP-infected HUVEC cells. Moreover, there was no induction of cell death in HL-7702 cells treated with the concentrated supernatant of LV-SP-TAT-apoptin/GFP-infected HUVEC cells. (Student's t-test, ${ }^{* *}$ P<0.05). (C) Using fluorescence microscopy, HepG2, Bel-7402 and HL-7702 cells treated with the concentrated supernatant of HUVEC cells infected with LV-SP-TAT-GFP for 30 min showed positive GFP expression in target cells. However, apoptin nuclear localization was detected only in HepG2 and Bel-7402 cells treated with the concentrated supernatant of HUVEC cells infected with LV-SP-TAT-apoptin for 30 min, while apoptin nuclear localization was not detected in HL-77002 cells treated with the concentrated supernatant of HUVEC cells infected with LV-SP-TAT-apoptin for 30 min. Magnification, x600.

disappeared within 2 weeks. The last tumor remained a steady size for a long period of time. Following dissection, H\&E staining revealed that it was a fibrous scar with a few HCC cells (Fig. 5B). These data strongly indicate that the LV-SPTAT-apoptin virus injection eradicated xenograft tumors. Lentivirus-based secretable TAT-apoptin eradicates HCC xenograft tumors in vivo after systemic delivery.

Toxicity of lentivirus-based secretable TAT-apoptin. To evaluate the possible toxic effects of LV-SP-TAT-apoptin, we injected 2.0x109 viral particles (LV-SP-TAT-apoptin, LV-SPTAT-GFP or saline) in healthy ICR mice via the tail vein. Since body weight is generally an accurate indicator of health status within age-matched groups, we used this parameter as a criterion of well-being. It can be seen in Fig. 5C that all groups gained weight similar to control animals (ranging within 10\%) after injection. After 1 week, all animals were sacrificed. Brain, lung, spleen, kidney, and liver samples were isolated for macroscopic evaluation and histological examination. No overt pathology was noted in these tissues (Fig. 5D). The general parameter measured (the weights of total body) were similar to those of the control groups at the end of the experiment (Fig. 5C). Based on our data, we conclude that significant
LV-SP-TAT-apoptin $\left(2 \times 10^{n}\right)$

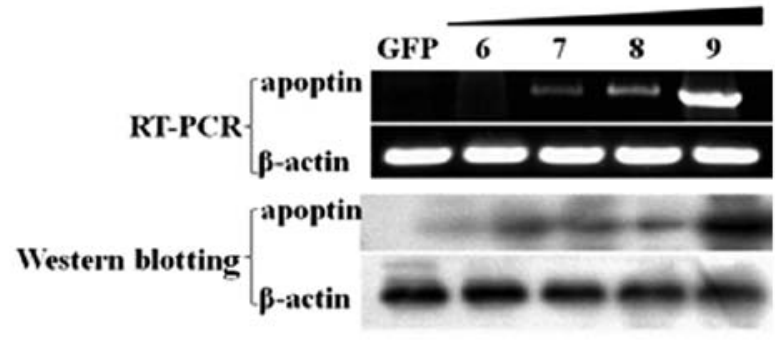

Figure 3. RT-PCR and western blotting results show a gradually increasing pattern in apoptin mRNA and protein levels, reflecting a dose-dependent expression of viral genes. The peak effect was seen at the dose of equal $2 \times 10^{9}$ particles per $50 \mathrm{~mm}^{3}$.

amounts of the lentivirus-based secretable TAT-apoptin can be administered without acute fatal toxicity.

\section{Discussion}

This report describes the generation of recombinant lentivirus expressing secretable TAT-apoptin and its effects on xeno- 
A

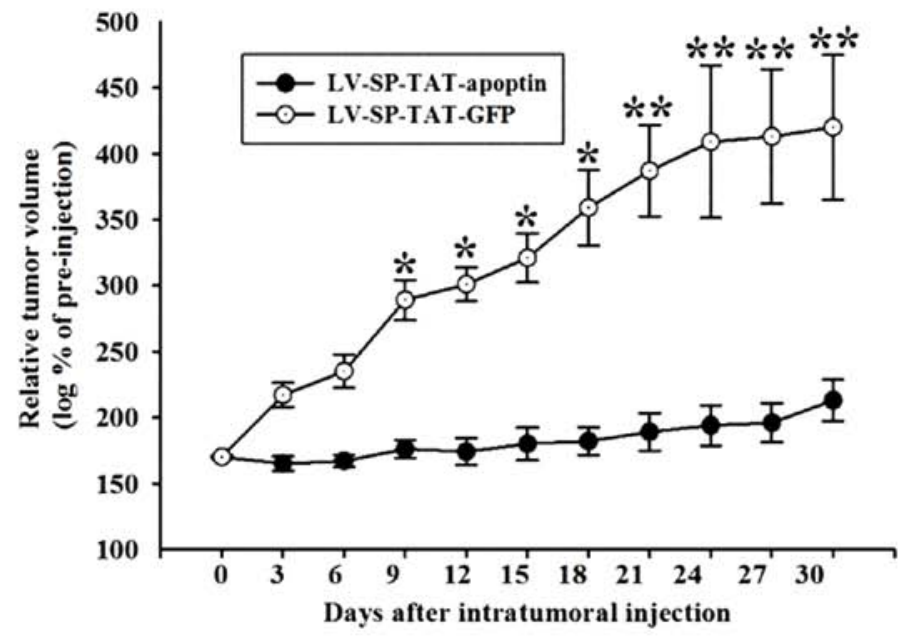

B

apoptin/GFP
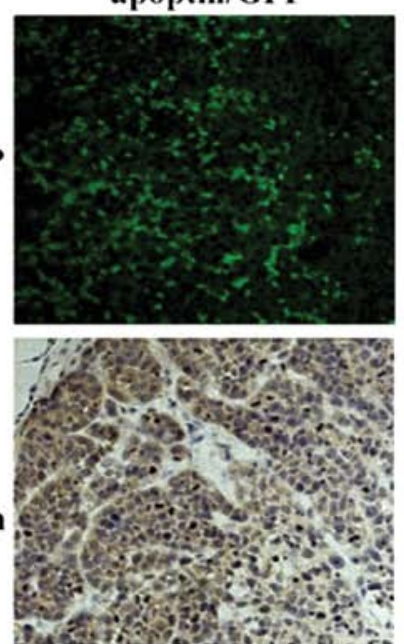

TUNEL
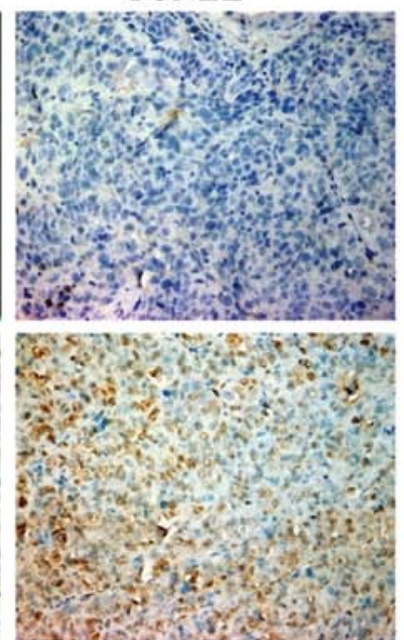

\section{LV-SP-TAT-apoptin}
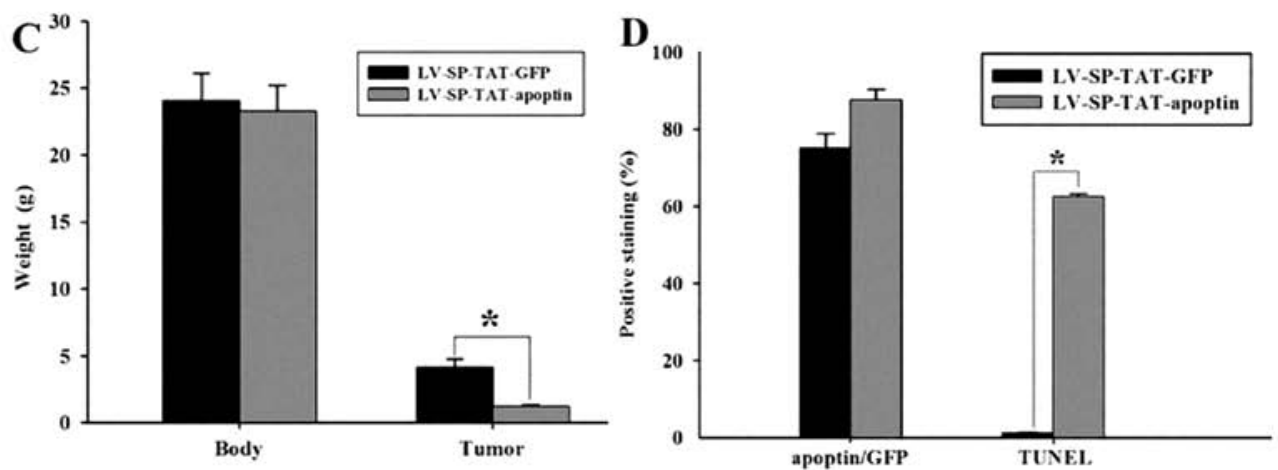

Figure 4. LV-SP-TAT-apoptin suppresses tumor growth in HepG2 xenografts. (A,C) Following intra-tumoral injection, LV-SP-TAT-GFP virus-injected xenograft tumors grew constantly. Conversely, LV-SP-TAT-apoptin virus-injected xenografts were completely suppressed. Quantitative data (mean \pm SEM) are presented in panels A, C. Representative results of two independent experiments (Student's t-test, ${ }^{*} \mathrm{P}<0.05 ;{ }^{* * *} \mathrm{P}<0.01$ ). (B) Apoptin/GFP expression was observed in virus-injected xenografts. Magnification, x400. (B, D) Higher apoptotic index (TUNEL assay) was found in LV-SP-TAT-apoptin virus-treated xenografts compared to LV-SP-TAT-GFP virus-treated xenografts. Quantitative data (mean \pm SEM) are presented in panel D. The asterisk indicates a significant difference compared to the control (Student's t-test, $\mathrm{P}<0.05$ ). Magnification, $\mathrm{x} 400$.

graft tumors in vivo. Our previous in vitro studies $(12,13)$, have shown that SP-TAT-apoptin induces apoptosis in HepG2 cells, but not in HUVEC cells. We expected, therefore, that the generation and production of recombinant lentivirus expressing the secretable SP-TAT-apoptin gene might greatly increase its potency once it is delivered in vivo for cancer therapy.
We report here that the resultant SP-TAT-apoptin/GFP was capable of being secreted from the producer cells and was taken up by several target cell lines. The efficient delivery of the secreted TAT-apoptin/GFP was demonstrated using both harvested culture supernatant as well as mixing experiments of the culture supernatant from producer cells and target cells. The TAT-apoptin/GFP was unable to enter the target cells 
A

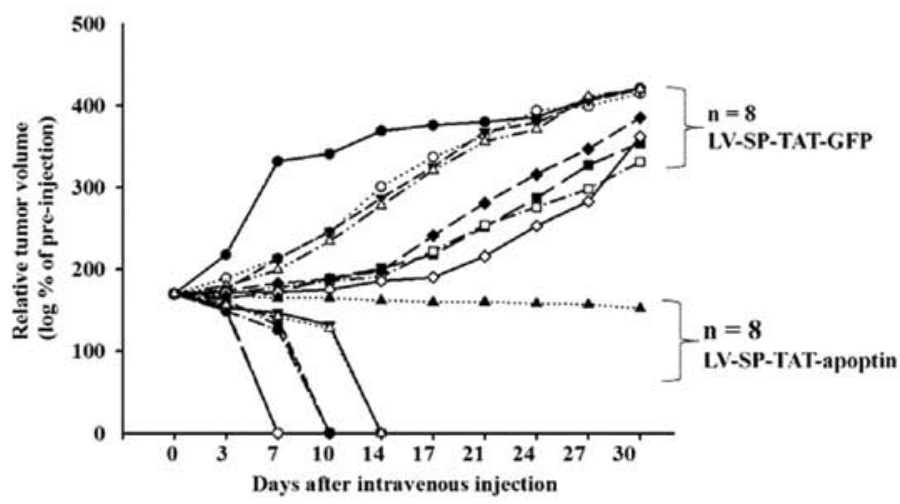

B

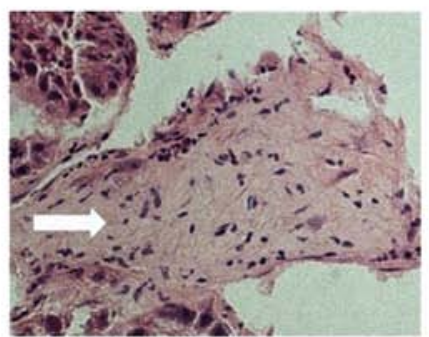

C

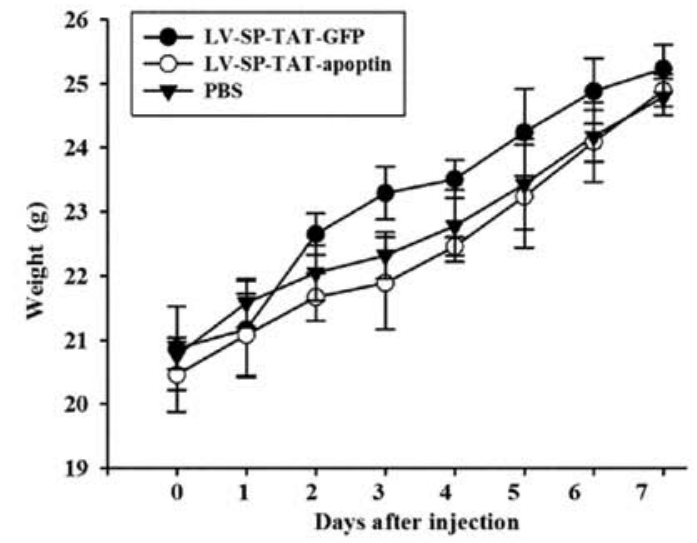

D
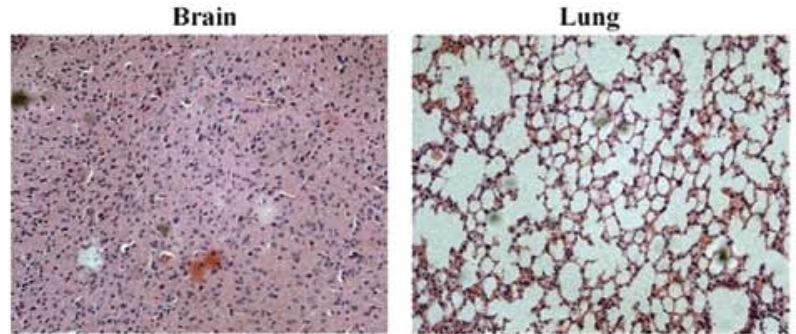

Kidney
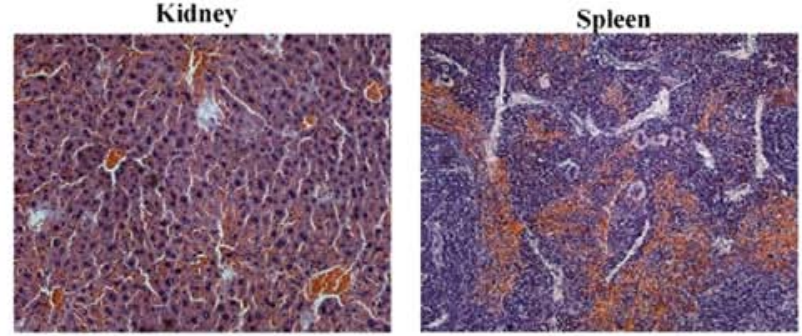

Figure 5. Systemic injection of LV-SP-TAT-apoptin virus eradicates HepG2 xenograft tumors in nude mice and the toxicity of LV-SP-TAT-apoptin in mice. (A) Data presented are the LV-SP-TAT-apoptin virus-injected relative tumor volume compared to the initial value from individual xenografts. Of note, 7 tumors disappeared within 2 weeks and the last one displayed as a steady node, whose image is shown in panels B, C following dissection. However, LV-SPTAT-GFP virus-injected tumor showed continuous growth. Quantitative data (mean \pm SEM) are presented in panel A. The asterisk indicates a significant difference compared to the control (Student's t-test, $\mathrm{P}<0.05$ ). (B) H\&E staining revealed that LV-SP-TAT-apoptin-injected tumor was a fibrous scar with a few HCC cells. Magnification, x200. Arrow, the fibrous scar. (C) Following virus injection, all groups gained weight similar to control animals (range within 10\%). Quantitative data (mean \pm SEM) are presented in panel A. (D) No overt pathology was noted in the lung, spleen, kidney, and liver tissues. Magnification, $\mathrm{x} 200$.

probably due to the fact that TAT-apoptin/GFP fusion protein without SP can be secreted into the culture supernatant. BFA, a drug that blocks secretion, prevents the secretion of SP-TATapoptin/GFP via the constitutive pathway. These results suggest that SP linked-TAT-apoptin/GFP is secreted through the constitutive pathway, but not TAT-apoptin/GFP.

To further assess the secretory ability of SP-TAT-apoptin/ GFP, lentivirus LV-SP-TAT-apoptin/GFP was generated as described in our previous publication (13). Our data showed the successful secretion of high levels of TAT-apoptin/GFP into the culture medium from HUVEC cells infected by LV-SPTAT-apoptin/GFP. Significantly, the secreted TAT-apoptin displaced to nucleus and induced cell death in target cancer cells. The next objective would be to test the efficacy of LV-SPTAT-apoptin infected cells to secrete this fusion protein and kill cancer cells within the tumor environment in vivo. There 
are two possible ways for the in vivo delivery of LV-SP-TATapoptin viruses: i) through intra-tumoral injection and ii) by injection via the tail vein. To mimic the clinical situation of cancer treatment, in our study, we first injected the LV-SP-TATapoptin viruses directly into existing xenograft tumors. Similar to other reports, this approach resulted only in the suppression of the xenograft tumor growth, and not in tumor elimination as we had expected, due to the uneven distribution of the locally injected viruses. After careful evaluation of the tumor section, we realized that the injected LV-SP-TAT-apoptin viruses were not evenly distributed inside the tumor mass. To achieve a more profound effect, we injected the LV-SP-TAT-apoptin viruses via the tail vein. Almost all the xenograft tumors disappeared following the treatment while the xenografts that received the control LV-SP-TAT-GFP viruses continued to grow, indicating a superior tumor-targeting efficiency through the systemic delivery.

Moreover, the animal studies presented in this paper demonstrate a low toxicity of SP-TAT-apoptin in vivo, confirming and extending the results of the in vitro studies. Healthy ICR mice were injected via the tail vein with LV-SPTAT-apoptin, LV-SP-TAT-GFP, and phosphate buffered saline (PBS), respectively. After 1 week, all animals were sacrificed. Lung, spleen, kidney, and liver samples were isolated for macroscopic evaluation and histological examination. No overt pathology was noted in these tissues. The general parameters measured (the weights of total body), were similar to those of the control groups at the end of the experiment. From these data, we conclude that significant amounts of the lentivirus-based secretable TAT-apoptin can be administered without acute fatal toxicity.

In this study, we demonstrated that systemic delivery of the lentivirus-based SP-TAT-apoptin viruses eradicated xenograft tumors without acute fatal toxicity. Our results indicate that secretable TAT-apoptin might be a potential strategy in hepatocellular carcinoma. However, as a clinical therapy for HCC, there are still a large number of issues to be resolved. Although lentivirus has been shown to be very efficient for delivering genes into cells, there are restrictions for its therapeutic use, such as risk of insertional mutagenesis and possible immunogenicity. Thus, side-effects from lentivirus-based SP-TAT-apoptin therapy may limit its application. Indeed, one of our future directions is to develop a novel delivery system combined with the secretable proteins to provide a safer and more effective therapeutic strategy. In summary, our data strongly suggest that systemic delivery of lentivirus-based secretable TAT-apoptin is feasible to eradicate liver cancer in vivo.

\section{Acknowledgements}

This study was supported by the National Natural Scientific Foundation of China (no. 81071692). We thank Professor Pei-jun Liu, Dr Lei Li, and Dr Lei Zhao for their helpful insight in the course of these studies and Professor Huang Chen for his technical advice.

\section{References}

1. Noteborn MH and van der Eb AJ: Apoptin-induced apoptosis: potential for antitumor therapy. Drug Resist Updat 1: 99-103, 1998.

2. Los M, Panigrahi S, Rashedi I, Mandal S, Stetefeld J, Essmann F, et al: Apoptin, a tumor-selective killer. Biochim Biophys Acta 1793: 1335-1342, 2009.

3. Russo A, Terrasi M, Agnese V, Santini D and Bazan V: Apoptosis: a relevant tool for anticancer therapy. Ann Oncol 17: vii115-23, 2006.

4. Alvisi G, Poon IK and Jans DA: Tumor-specific nuclear targeting: promises for anti-cancer therapy? Drug Resist Updat 9: 40-50, 2006.

5. Maddika S, Mendoza FJ, Hauff K, Zamzow CR, Paranjothy T and Los M: Cancer-selective therapy of the future: apoptin and its mechanism of action. Cancer Biol Ther 5: 10-19, 2006.

6. Poon IK, Oro C, Dias MM, Zhang JP and Jans DA: A tumor cell-specific nuclear targeting signal within chicken anemia virus VP3/apoptin. J Virol 79: 1339-1341, 2005.

7. Chauhan A, Tikoo A, Kapur AK and Singh M: The taming of the cell penetrating domain of the HIV Tat: myths and realities. J Control Release 117: 148-162, 2007.

8. Dietz GP and Bahr M: Delivery of bioactive molecules into the cell: the Trojan horse approach. Mol Cell Neurosci 27: 85-131, 2004.

9. Wang H, Zhong CY, Wu JF, Huang YB and Liu CB: Enhancement of TAT cell membrane penetration efficiency by dimethyl sulphoxide. J Control Release 143: 64-70, 2010.

10. Flinterman M, Farzaneh F, Habib N, Malik F, Gaken J and Tavassoli M: Delivery of therapeutic proteins as secretable TAT fusion products. Mol Ther 17: 334-342, 2009.

11. Guelen L, Paterson H, Gaken J, Meyers M, Farzaneh F and Tavassoli M: TAT-apoptin is efficiently delivered and induces apoptosis in cancer cells. Oncogene 23: 1153-1165, 2004.

12. Han SX, Ma JL, Lv Y, Huang C, Liang HH and Duan KM: Secretory Transactivating Transcription-apoptin fusion protein induces apoptosis in hepatocellular carcinoma HepG2 cells. World J Gastroenterol 14: 3642-3649, 2008.

13. Han SX, Zhao J, Ma JL, Huang C, Lu Y, Ou W, et al: The effect of the fused gene of SP-TAT-Apoptin transfected by lentivirus on HepG2 cells. Xi Bao Yu Fen Zi Mian Yi Xue Za Zhi 26: 310-312, 2010 (In Chinese).

14. Barash S, Wang W and Shi Y: Human secretory signal peptide description by hidden Markov model and generation of a strong artificial signal peptide for secreted protein expression. Biochem Biophys Res Commun 294: 835-842, 2002.

15. Lippincott-Schwartz J, Yuan LC, Bonifacino JS and Klausner RD: Rapid redistribution of Golgi proteins into the ER in cells treated with brefeldin A: evidence for membrane cycling from Golgi to ER. Cell 56: 801-813, 1989.

16. Pelletier L, Jokitalo E and Warren G: The effect of Golgi depletion on exocytic transport. Nat Cell Biol 2: 840-846, 2000. 